\section{The Effect of High-Frequency Repetitive Transcranial Magnetic Stimulation on Functional Indices of Affected Upper Limb in Patients with Subacute Stroke}

\author{
Farzaneh Moslemi Haghighi, ${ }^{1,2}$, Amin Kordi Yoosefinejad ${ }^{1,3}$, Mohsen \\ Razeghi ${ }^{1,3}{ }^{\oplus *}$, Abdolhamid Shariat ${ }^{4,5}$, Zahra Bagheri ${ }^{6}$, Katayoon Rezaei ${ }^{1,2}$
}

\begin{abstract}
Background: Repetitive transcranial magnetic stimulation (rTMS) is a novel technique that may improve recovery in patients with stoke, but the role of rTMS as an applied and practical treatment modality for stroke rehabilitation has not been established yet.
\end{abstract}

Objective: This study was conducted to determine the effects of a rehabilitation program (RP) in conjunction with rTMS on functional indices of the paretic upper limb in the subacute phase of stroke.

Material and Methods: In this experimental study, twenty patients in the subacute phase of stroke were randomly assigned into two groups: The high frequency rTMS (HF-rTMS) in conjunction with RP (experimental group), and the RP group (control group). The experimental group received 10 sessions of $20 \mathrm{~Hz}$ rTMS on the affected primary motor cortex and the other group received 10 sessions of RP. In experimental group, RP for the paretic hand was conducted following rTMS session. Box and block test (BBT), Fugl-Meyer Motor Assessment for upper limb (FMA-UL), grip strength and pinch strength were used to assess motor function before the first session and after the last session of treatment.

Results: Significant improvement in BBT, FMA-UL, grip strength and pinch strength was observed in both groups. Improvement of BBT and grip strength was significantly greater in the experimental group rather than the control group $(p<0.05)$. FMA-UL score and the pinch strength were greater in the experimental group, although the differences were not statistically significant.

Conclusion: HF-rTMS in conjunction with RP is effective to improve the function of upper limb. It seems HF-rTMS is a novel feasible and safe technique for hemiparesis patients in the subacute phase of stroke.

Citation: Moslemi Haghighi F, Kordi Yoosefinejad A, Razeghi M, Shariat A, Bagheri Z, Rezaei K. The Effect of High-Frequency Repetitive Tran-

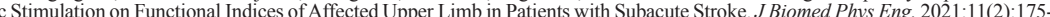
184. doi: 10.31661 /jbpe.v0i0.879

\section{Keywords}

Transcranial Magnetic Stimulation; Stroke; Hand Function; Motor Recovery; Rehabilitation

\section{Introduction}

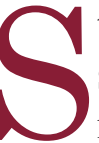
troke is a principal cause of severe disability in adults [1]. After stroke, approximately $80 \%$ of survivors experience motor impairment that declines the quality of their daily lives [2-4]. Hemiparesis or muscle weakness on the contralateral side to the affected cerebral hemisphere is the most obvious motor disability after the stroke [3]. According to Twitchel's theory, upper limb deficits following stroke
${ }^{1} \mathrm{PhD}$, Department of Physiotherapy, School of Rehabilitation Sci-

ences, Shiraz University of Medical Sciences,

Shiraz, Iran

${ }^{2} \mathrm{PhD}$, Student Research

Committee, School of

Rehabilitation Sciences,

Shiraz University of

Medical Sciences, Shi-

raz, Iran

${ }^{3} \mathrm{PhD}$, Rehabilitation

Sciences Research

Center, Shiraz University

of Medical Sciences,

Shiraz, Iran

${ }^{4} \mathrm{MD}$, Clinical Neurology

Research Center, Shiraz

University of Medical Sci-

ences, Shiraz, Iran

${ }^{5} \mathrm{MD}$, Neuroscience

Research Center, Shiraz

University of Medical Sci-

ences, Shiraz, Iran

${ }^{6} \mathrm{PhD}$, Department of

Biostatistics, School of

Medicine, Shiraz Univer-

sity of Medical Sciences,

Shiraz, Iran

*Corresponding author:

Mohsen Razeghi

Professor, Department

of Physiotherapy, School of Rehabilitation Sci-

ences, Shiraz University

of Medical Sciences,

Shiraz, Iran

E-mail: razeghm@sums. ac.ir

Received: 17 December 2017 Accepted: 28 March 2018 
are more than those of the lower limb and the improvement of upper limb shows more delay and less obtained recovery [5]. Nearly $60-70 \%$ of the patients experience upper limb impairments on the hospital admission, with approximately half (33\% of all strokes) classified as having severe paresis [6-8]. Two to four years after stroke, $50-70 \%$ of those surviving from stroke have lost the function in paretic upper limb [8,9]. Rehabilitation has an important role in improving the function of paretic limb. Neuroimaging studies have exhibited that rehabilitation might induce brain plasticity after stroke [10-12]. Recently, the studies have focused on novel interventions to make a recovery from the upper extremity in patients with stroke.

Several studies reported that rTMS had positive effects on treating patients with depression, Parkinson's disease, Huntington's disease, dystonia and cerebral palsy [13-16]. Recently, rTMS has been used in post- stroke care $[17,18]$. The rTMS has an important role in stroke care, including diagnosis, prognosis, and intervention [19]. The rTMS induces depolarization and production of action potential in the stimulated area of cerebral cortex. Based on the characteristics of stimulation, such as frequency, coil orientation, and intensity of stimulation, rTMS can alter the excitability of corticospinal pathways and induce lasting effects on cortical neuroplasticity. Although the neural mechanisms of rTMS on the motor recovery after stroke are not clear, it is vastly suggested that rTMS can influence synaptic connections and produce long-term potentiation and long-term depression [20].

The effect of rTMS on motor recovery after stroke is based on the inter-hemispheric competition model [21]. According to this model, the contralesional hemisphere produces extreme inhibition on the lesional hemisphere, which may lead to reduced excitability and the cortical drive to the paretic extremity. Neuroimaging researches recognized that excitatory high-frequency rTMS of the lesioned hemi- sphere and inhibitory low-frequency rTMS of the unlesioned hemisphere can affect the control of cerebral cortex activation and can regulate cortical excitability between the two hemispheres [22].

Despite several studies on patients with stroke, the usefulness of combining rTMS with rehabilitation treatment is unclear. More studies are needed to clarify the most beneficial effects of rTMS and to certify practical effects of rTMS as a neurorehabilitation tool in treatment of patients with stroke. It might be possible that the use of rTMS prior to rehabilitation training could increase the therapeutic effects and decrease the treatment periods.

According to the previous studies, the subacute stage of stroke is the most proper time period for neurorehabilittion. In this stage, natural adaptations and functional recovery occur quickly on a wide scale [23, 24]. According to this view point, combining rTMS and rehabilitation treatment in the subacute stage might produce a more powerful effect on functional motor recovery in patients with paretic upper limb. The purpose of this study was to determine the effects of a RP in conjunction with rTMS on various functions of lesioned upper limb in the subacute phase of stroke. It was hypothesized that the combination of rTMS and RP could accelerate the functional recovery and enhance the effect of rehabilitation treatment in patients with subacute stroke.

\section{Material and Methods}

\section{Subjects}

Twenty survivors of stroke with motor deficits in upper limb (9 women, 11 men) voluntarily participated in this experimental study. The sample size was determined according to the results of a previous study that investigated the effect of rTMS in subacute stroke patients with motor deficits [25]. Considering a power of $80 \%$ and a critical alpha level of $0.05,12$ patients (six in each group) were 
Effect of Transcranial Magnetic Stimulation on Upper-limb Strok

determined to detect group differences. To account for possible dropouts, we added 8 more subjects. Therefore, 20 patients were entered into the study. The inclusion criteria were: (a) first-ever unilateral stroke, (b) ischemic or hemorrhagic subcortical lesion within the territory of the middle cerebral artery confirmed by magnetic resonance imaging and a professional neurologist, (c) the age from 30 to 65 years, (d) the post-onset duration of less than 6 months (e) Fugl-Meyer Motor Assessment for upper limb (FMA-UL) score of 22-44. The exclusion criteria for participants were as follows: (a) the past history of epilepsy or seizure, (b) the use of cardiac pacemaker or medical pumps, (c) cognitive impairments ( $>25$ in mini- mental function measure), (d) intracranial metal implants, (e) metal objects in the eye, (f) previous orthopedic problems in the upper extremity, (g) the history of other neurologic diseases, (h) pregnancy, and (i) severe aphasia. A written informed consent was signed by all the participants before the beginning of the interventions. All study protocols were reviewed and approved by local ethic committee (No: CT-94-7605).

\section{Study design}

This study was a randomized, parallel design, double-blind controlled trial. Patients were assigned into two groups; the rTMS in conjunction with RP group (experimental group) and the rehabilitation group (the control group). All the participants were blinded to a treatment allocation and treated by an experienced physiotherapist. Each subject took 10 treatment sessions, three times per week. Clinical measures were evaluated by a welltrained physiotherapist blinded to the subjects' group allocation during two separate sessions: a day before the first intervention session and one day after the last session.

\section{Assessments}

The BBT, FMA-UL, grip strength and pinch strength were selected as the outcome mea- sure of paretic upper limb motor recovery. The BBT is a valid and reliable test to estimate unilateral gross manual dexterity [26]. It is comprised of a partitioned box and 150 blocks. During this brief performance-based test, subjects must pass as many blocks as possible from one box to another one in a minute. A total amount of blocks that subject could pass correctly reveal the gross dexterity of the upper extremity [27]. The FMA-UL was used to assess the paretic upper limb motor function. Reliability and validity of this test have been established previously [28]. This assessment includes 33 quantitative tasks and each task is graded from zero (no activity) to two (complete activity). The total score (66 points) indicates the normal motor function of upper extremity [29, 30].

A handheld grip dynamometer (Model SH5001, SEHAN, and Masan, Korea) and a hydraulic pinch gauge (Model SH5005, SEHAN, and Masan, Korea) were used to assess maximal voluntary isometric grip strength and pinch strength, respectively. To measure grip and pinch strength, the elbow was held at 90 degrees of flexion and the forearm was kept in midposition. Subjects applied a maximal effort on the instruments for a period of five seconds. Three trials were done with a twominute rest between them. The mean value of the three trials was recorded.

\section{Procedure}

Patients in the control group were treated with RP for 10 sessions, 3 times a week. RP was carried out by the same physiotherapist. According to the severity of hemiparesis and motor deficits, RP consisted of a range of motion training, motor control education, the stretch of hypertonic muscles, the practice of activities of daily living, and functional electrical stimulation (for 20 minutes, with $25 \mathrm{~Hz}$ frequency and 0-250 $\mu \mathrm{sec}$ pulse wide to obtain controlled muscle contraction).

The patients in the experimental group received HF-rTMS in conjunction with RP. For 
the HF-rTMS protocol, this study used a Magstim Rapid stimulation (SM9000, Neurosoft, Russia) and a $100 \mathrm{~mm}$ figure-of-eight coil. Each Patient seated in a comfortable reclining position and wore a cap with equal marks 1-cm apart. The coil was positioned tangentially to the scalp over the hand area of the ipsilesional primary motor cortex (M1), with the handle orienting 45 degrees postro-laterally, on the hotspot of the affected abductor pollicis bravis (APB). To identify the APB hot spot, the handle was moved forward at $1-\mathrm{cm}$ increments. The optimal stimulation site (hot spot) was determined as the point where the stimulation of suprathreshold intensity induced the largest motor evoked potential (MEP) in the APB muscle. Electromyographic (Neurowerk EMG, SIGMA Medizin-Techik, Germany) activity was recorded from $\mathrm{Ag}-\mathrm{Ag}-\mathrm{Cl}$ electrodes placed in the belly-tendon technique on the skin overlying the APB, and the signal was amplified, filtered $(20 \mathrm{~Hz}-15 \mathrm{KHz})$ and digitized at a sampling rate of $50 \mathrm{kHz}$ for offline analysis. The hot spot of APB was marked on the cap with a marker to ensure the uniform coil position. The resting motor threshold (rMT) was determined in each subject prior to the rTMS procedure and defined as the minimum output of stimulation that was able to elicit MEP at least $50 \mu \mathrm{v}$ in at least 5 of 10 consecutive trials. rTMS system was set on 20 $\mathrm{Hz}$ frequency, 5 seconds duration, 50 seconds inter- train interval, 20 train , and 90\% of rMT (total 2000 pulse/day). The rTMS protocol used in the present study was performed according to the safety guidelines for rTMS applications [31]. RP was performed following the rTMS protocol in each session.

\section{Statistical analysis}

Statistical analyses were done using SPSS version 23 (IBM statistics, New York, USA). The Kolmogorov- Smirnov test was used to assess the normal distribution of data. In each group, differences between pre-test and posttest values were analyzed using Wilcoxon signed ranks test. The Mann-Whitney-U was used to evaluate between-group differences. The level of statistical significance for all analyses was set at $\mathrm{p}<0.05$.

\section{Results}

The treatment and assessment sessions were completed by all the participants. The subjects' clinical characteristics and demographic data in two groups are shown in Table 1. There were no significant differences between the two groups in both demographic and clinical characteristics at the baseline. The clinical outcome measured before and after the interventions are depicted in Table 2. All clinical measurements improved significantly during 10 sessions in both groups. The extend of improvement in BBT for upper limb was significantly larger in the experimental group than in the control group $(6.50 \pm 2.67$ units in the HF-rTMS group, $2.30 \pm 1.63$ units in the control group, $\mathrm{p}=0.003$ ) (Figure 1). Similarly, the improvement in grip strength was also significantly larger in the HF-rTMS group than in the control group $(3.07 \pm 0.86 \mathrm{~kg}$ in the HF-rTMS group, $1.37 \pm 1.66 \mathrm{~kg}$ in the control group, $\mathrm{p}=0.007$ ) (Figure 2). The improvement in FMA-UL and pinch strength was also larger in the HF-rTMS group compared to the control group; otherwise the differences were not statistically significant (FMA-UL: $9.60 \pm 4.27$ scores in the HF-rTMS group, $6.60 \pm 5.01$ scores in the control group, $\mathrm{p}=0.063$; pinch strength: $1.12 \pm 0.66 \mathrm{~kg}$ in the HF-rTMS group, $0.77 \pm 0.62 \mathrm{~kg}$ In the control group, $\mathrm{p}=0.353$ ) (Figures 3 and 4).

\section{Discussion}

All participants completed the study protocol without any adverse events. In addition, the improvement of BBT and grip strength scores was significantly more obvious in the experimental group rather than the control group. Therefore, HF- rTMS had positive effects on improving gross dexterity and grip strength of the paretic hand in subacute phase 
Table 1: Demographic data and clinical characteristics in two groups $(n=20)$.

\begin{tabular}{lccc}
\multicolumn{1}{c}{ Parameters } & Control Group (n=10) & $\begin{array}{c}\text { HF-rTMS Group } \\
(\mathbf{n = 1 0 )}\end{array}$ & p-value \\
\hline Age (year) & $53.90 \pm 13.06$ & $50.50 \pm 9.47$ & ${ }^{*} 0.144$ \\
\hline Gender, male/ female, $\mathrm{n}(\%)$ & $5(50) / 5(50)$ & $6(60) / 4(40)$ & ${ }^{\dagger} 0.653$ \\
\hline Time since stroke (month) & $3.20 \pm 1.68$ & $3.00 \pm 1.41$ & ${ }^{*} 0.516$ \\
\hline Dominant side, right/ left, $\mathrm{n}(\%)$ & $10(100) / 0(0)$ & $8(80) / 2(20)$ & ${ }^{\dagger} 0.136$ \\
\hline Paretic side, right/ left, $\mathrm{n}(\%)$ & $5(50) / 5(50)$ & $4(40) / 6(60)$ & ${ }^{\dagger} 0.653$ \\
\hline FMA-UL(score) & $36.7 \pm 7.71$ & $30.5 \pm 6.78$ & ${ }^{*} 0.278$ \\
\hline BBT (unit) & $23.2 \pm 16.47$ & $13.5 \pm 10.9$ & ${ }^{*} 0.313$ \\
\hline Grip strength $(\mathrm{kg})$ & $4.67 \pm 3.89$ & $5.27 \pm 2.39$ & ${ }^{*} 0.622$ \\
\hline Pinch strength $(\mathrm{kg})$ & $5.44 \pm 2.97$ & $2.55 \pm 1.33$ & ${ }^{*} 0.823$
\end{tabular}

FMA-UE: upper limb of Fugl-Meyer Assessment; BBT: Box and Block Test; HF-rTMS: High frequency repetitive transcranial magnetic stimulation.

Values are expressed as mean \pm standard deviation or frequency.

*p-value based on Mann-Whitney; ${ }^{\dagger}$-value based on Chi-square

Table 2: Clinical variables of the lesioned upper limb at pre- and post- intervention in both groups.

\begin{tabular}{ccccccc} 
& \multicolumn{2}{c}{ Control Group (n=10) } & \multicolumn{2}{c}{ HF-rTMS Group (n=10) } \\
\cline { 2 - 6 } & Pre M (SD) & Post M (SD) & p-value & Pre M (SD) & Post M (SD) & p-value \\
\hline FMA-UL(score) & $36.7(7.71)$ & $43.3(10.28)$ & 0.005 & $30.50(6.78)$ & $40.10(9.49)$ & 0.005 \\
\hline BBT(unit) & $23.20(16.47)$ & $25.50(17.50)$ & 0.007 & $13.50(10.91)$ & $20.00(10.29)$ & 0.005 \\
\hline Grip strength (kg) & $4.67(3.89)$ & $6.04(4.21)$ & 0.026 & $5.27(2.39)$ & $8.35(2.86)$ & 0.005 \\
\hline Pinch strength (kg) & $2.97(1.38)$ & $3.74(1.58)$ & 0.018 & $2.55(1.33)$ & $3.67(1.79)$ & 0.005
\end{tabular}

FMA-UL: upper limb of Fugl-Meyer Assessment; BBT: Box and Block Test; HF-rTMS: High frequency repetitive transcranial magnetic stimulation; $\mathrm{M}(\mathrm{SD})$ : Mean \pm standard deviation.

Significance level for within group comparisons.

of stroke.

Applying HF-rTMS over the lesional hemisphere has been reported to enhance the motor function of the paretic upper extremity following chronic phase of stroke. Yozbatiran et al. showed that a single session of HF-rTMS (20 $\mathrm{Hz}$ ) had a beneficial effect on the motor arm function in the chronic phase of stroke [32]. Kim et al. reported that a single session of HF-rTMS $(10 \mathrm{~Hz})$ could improve motor learn- ing and facilitate practice-dependent plasticity in patients with chronic stroke [33]. Ji et al. showed that the use of HF-rTMS $(10 \mathrm{~Hz})$ on the ipsilesional hemisphere in conjunction with mirror therapy could improve hand dexterity and movement speed in patients with chronic stroke [34]. Similarly, Rajak et al. reported that 20 sessions of $5 \mathrm{~Hz}$ or $10 \mathrm{~Hz}$ rTMS at ipsilesional M1 in conjunction with physiotherapy improved significantly the hand 


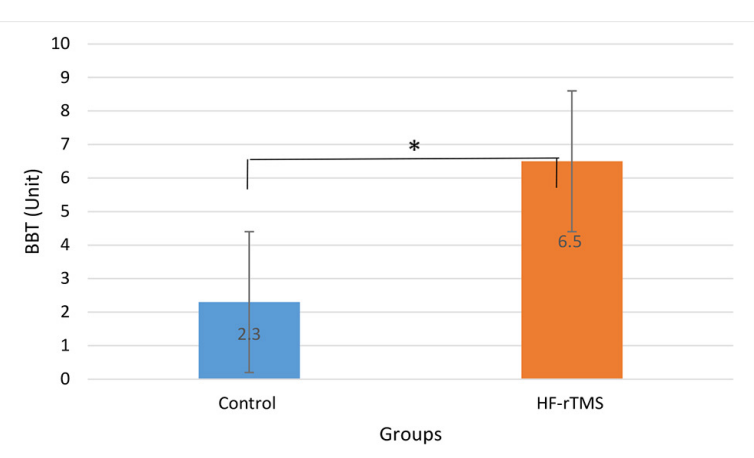

Figure 1: Comparison of mean difference of Box and Block Test (BBT) between two groups

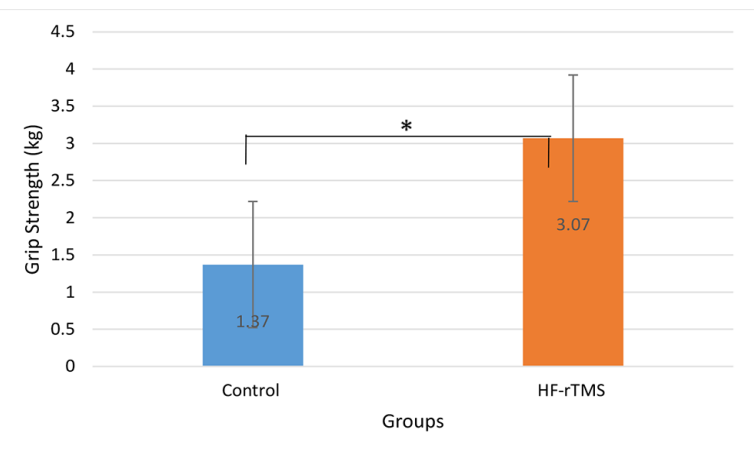

Figure 2: Comparison of mean difference of grip strength between two groups

function in children with spastic cerebral palsy [35].

The neural mechanisms of motor recovery following rTMS intervention are not well understood.

There was evidence suggesting that rTMS can cause plastic changes in the microstructure of white matter and gray matter in the central nervous system [1]. The increase of growth factors such as brain-derived neurotrophic factor (BDNF) has been reported after the use of rTMS [36, 37]. Animal studies suggested that rTMS can enhance angiogenesis and increase cerebral blood flow in paretic adult rats. It has been suggested that rTMS can reduce the extent of infarction and neural death via the upregulation of anti-apoptosis of cells located at the margin of the lesioned area [38-41]. Previous studies have shown that multi-session

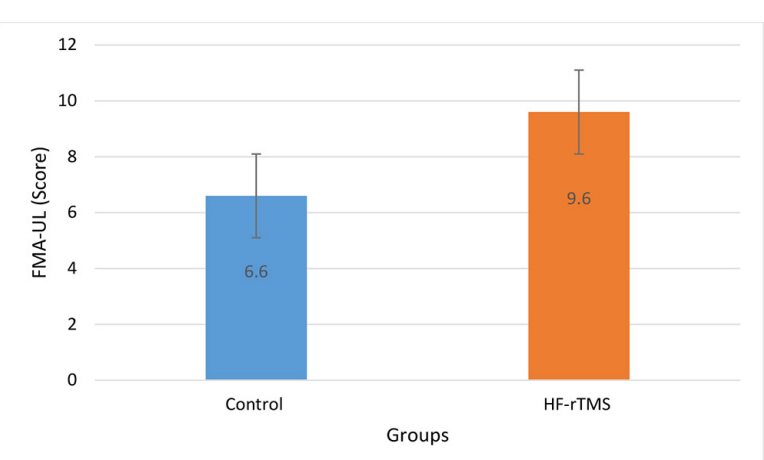

Figure 3: Comparison of mean difference of Fugl-Meyer Motor Assessment for upper $\operatorname{limb}($ FMA-UL) between two groups

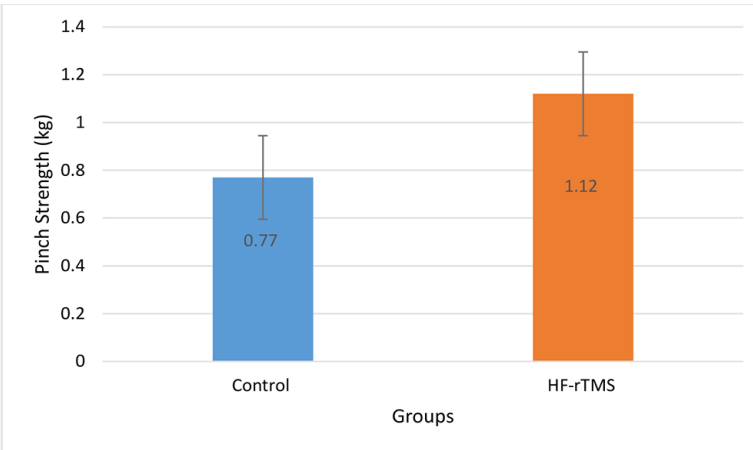

Figure 4: Comparison of mean difference of pinch strength between two groups

rTMS treatment provides a long-lasting effect on cortical excitability and induces long-term potentiation [42].

Data from animal models showed that rehabilitation and exercise training can change neural architecture, such as neural sprouting, synaptogenesis and dendritic branching [4347]. Furthermore, exercise training can activate molecular pathways that are important not only for neurogenesis but also for learning and memory [48]. A recent study showed that patients with mild-moderate levels of disabilities can benefit from using exercise and practice training that activate neural and muscular mechanisms of activity-dependent plasticity and learning [49]. In line with the previous study, our finding confirmed that the combination of HF-rTMS and RP could accelerate hand dexterity and grip strength of paretic 
hand in patients with subacute stroke.

Our finding showed that the improvement of FMA-UL and pinch strength of the impaired upper limb was greater in the experimental group than the control group, although both groups showed significant functional improvements. Despite the previous studies reported that the application of rTMS with various frequency could enhance FMA-UL scale $[25,34]$, in the present study no significant differences were seen between two groups. Kim et al. reported that 10 sessions of $20 \mathrm{~Hz}$ rTMS at ipsilesional motor cortex significantly improved FMA-UL of the affected upper limb, but in their study the significant improvement of lateral pinch was not seen [25]. In their study, each subject received 10 treatment sessions over 2 weeks versus 3 weeks in our study. It seems that not only different characteristics of stimulation, such as frequency, coil orientation, and intensity influence the effect of rTMS but also the number of sessions and inter- session interval may alter the efficacy of rTMS on the motor recovery in stoke. Further study is needed to clarify the most beneficial sessions and inter-session interval of rTMS. Moreover, the accurate parameters administering the rTMS are not well known and effects may vary extremely while depending on interindividual variability such as the size and location of the brain lesions and the severity of the motor deficits. Although the result of this and previous researches are preliminary taken together, they provide new information and instruction about the potential therapeutic usefulness of HF-rTMS for functional recovery following stroke.

Generally, our finding revealed that HF-rTMS in conjunction with RP is more effective to improve the function of upper limb, than RP alone. It seems HF-rTMS is a novel feasible and safe technique for hemiparesis patients with subacute stroke.

This study had some limitations. The first, we treated the patients with $22-44$ points in FMA-UL. Therefore, the effect cannot be gen- eralized about patients with greater deficits. The second, we used a technique of RP that is a common approach in rehabilitation clinics, but the effect of rTMS in conjunction with other rehabilitation techniques (such as proprioceptive neuromuscular facilitation, constraint-induced movement therapy, bilateral arm training and mental practice) is unknown. Future studies should involve other rehabilitation protocols in conjunction with rTMS. Since, we didn't verify the persistence of the longterm effects, it is necessary to perform similar studies on patients with follow-up evaluation.

\section{Conclusion}

The combination of HF-rTMS and RP is a safe and practical intervention for hemiparesis patients with subacute stroke. To improve upper limb hemiparesis in such patients, HFrTMS seems to be more beneficial than the rehabilitation treatment, alone, thus further studies should investigate the influence of other specific rehabilitation techniques on the response to rTMS. Our results suggested that the number of sessions and inter- session intervals were important parameters that should be considered in rTMS interventions in patients with stroke.

\section{Acknowledgment}

This study was extracted from the Ph.D. thesis inscribed by Farzaneh Moslemi Haghighi and was financially supported by Shiraz University of Medical Sciences. Grant No: 947605 .

\section{Conflict of Interest}

None

\section{References}

1. Guo Z, Jin Y, Peng H, Xing G, Liao X, Wang Y, et al. Ipsilesional High Frequency Repetitive Transcranial Magnetic Stimulation Add-On Therapy Improved Diffusion Parameters of Stroke Patients with Motor Dysfunction: A Preliminary DTI Study. Neural Plast. 2016;2016:6238575. doi: 10.1155/2016/6238575. PubMed PMID: 27840742. PubMed PMCID: 
PMC5093297.

2. Buma FE, Lindeman E, Ramsey NF, Kwakkel G. Functional neuroimaging studies of early upper limb recovery after stroke: a systematic review of the literature. Neurorehabil Neural Repair. 2010;24:589608. doi: $10.1177 / 1545968310364058$. PubMed PMID: 20439501.

3. Langhorne P, Coupar F, Pollock A. Motor recovery after stroke: a systematic review. Lancet Neurol. 2009;8:741-54. doi: 10.1016/S14744422(09)70150-4. PubMed PMID: 19608100.

4. Urton ML, Kohia M, Davis J, Neill MR. Systematic literature review of treatment interventions for upper extremity hemiparesis following stroke. Occup Ther Int. 2007;14:11-27. doi: 10.1002/oti.220. PubMed PMID: 17623376.

5. Twitchell TE. The restoration of motor function following hemiplegia in man. Brain. 1951;74:443-80. PubMed PMID: 14895765.

6. Nakayama H, Jorgensen HS, Raaschou HO, Olsen TS. Recovery of upper extremity function in stroke patients: the Copenhagen Stroke Study. Arch Phys Med Rehabil. 1994;75:394-8. PubMed PMID: 8172497.

7. Stig Jørgense H, Nakayama H, Otto Raaschou H, Møller Pedersen P, Houth J, Skyhøj Olsen T. Functional and neurological outcome of stroke and the relation to stroke severity and type, stroke unit treatment, body temperature, age, and other risk factors: The Copenhagen Stroke Study. Top Stroke Rehabil. 2000;6:1-19. doi: 10.1310/bt7j-2n6uvd53-e1qu.

8. Wade DT, Langton-Hewer R, Wood VA, Skilbeck $\mathrm{CE}$, Ismail HM. The hemiplegic arm after stroke: measurement and recovery. I Neurol Neurosurg Psychiatry. 1983;46:521-4. PubMed PMID: 6875585. PubMed PMCID: PMC1027442.

9. Broeks JG, Lankhorst GJ, Rumping K, Prevo AJ. The long-term outcome of arm function after stroke: results of a follow-up study. Disabil Rehabil. 1999;21:357-64. PubMed PMID: 10503976.

10. Calautti C, Baron JC. Functional neuroimaging studies of motor recovery after stroke in adults: a review. Stroke. 2003;34:1553-66. doi: 10.1161/01. STR.0000071761.36075.A6. PubMed PMID: 12738893.

11. Johansen-Berg H, Dawes H, Guy C, Smith SM, Wade DT, Matthews PM. Correlation between motor improvements and altered fMRI activity after rehabilitative therapy. Brain. 2002;125:2731-42. PubMed PMID: 12429600.

12. Liepert J, Bauder $\mathrm{H}$, Wolfgang HR, Miltner WH, Taub E, Weiller C. Treatment-induced cortical reorganization after stroke in humans. Stroke. 2000;31:1210-6. PubMed PMID: 10835434.

13. Kamble N, Netravathi M, Pal PK. Therapeutic applications of repetitive transcranial magnetic stimulation (rTMS) in movement disorders: a review. Parkinsonism Relat Disord. 2014;20:695-707. doi: 10.1016/j.parkreldis.2014.03.018. PubMed PMID: 24726453.

14. Gupta M, Rajak B, Bhatia D, Mukherjee A. Transcranial magnetic stimulation therapy in spastic cerebral palsy children improves motor activity. J Neuroinfect Dis. 2016;7:2.

15. Yang YR, Tseng CY, Chiou SY, Liao KK, Cheng SJ, Lai $\mathrm{KL}$, et al. Combination of rTMS and treadmill training modulates corticomotor inhibition and improves walking in Parkinson disease: a randomized trial. Neurorehabil Neural Repair. 2013;27:79-86. doi: 10.1177/1545968312451915. PubMed PMID: 22785003.

16. Kimberley TJ, Schmidt RL, Chen M, Dykstra DD, Buetefisch CM. Mixed effectiveness of rTMS and retraining in the treatment of focal hand dystonia. Front Hum Neurosci. 2015;9:385. doi: 10.3389/ fnhum.2015.00385. PubMed PMID: 26217209. PubMed PMCID: PMC4496570.

17. Kubis N. Non-Invasive Brain Stimulation to Enhance Post-Stroke Recovery. Front Neural Circuits. 2016;10:56. doi: 10.3389/fncir.2016.00056. PubMed PMID: 27512367. PubMed PMCID: PMC4961863.

18. Takeuchi N. Overview of non-invasive brain stimulation for motor recovery after stroke. Int $J$ Phys Med Rehabil. 2013;1:2. doi: 10.4172/23299096.1000114.

19. Dimyan MA, Cohen LG. Contribution of transcranial magnetic stimulation to the understanding of functional recovery mechanisms after stroke. Neurorehabil Neural Repair. 2010;24:125-35. doi: 10.1177/1545968309345270. PubMed PMID: 19767591. PubMed PMCID: PMC2945387.

20. Fitzgerald PB, Fountain S, Daskalakis ZJ. A comprehensive review of the effects of rTMS on motor cortical excitability and inhibition. Clin Neurophysiol. 2006;117:2584-96. doi: 10.1016/j. clinph.2006.06.712. PubMed PMID: 16890483.

21. Murase N, Duque J, Mazzocchio R, Cohen LG. Influence of interhemispheric interactions on motor function in chronic stroke. Ann Neurol. 2004;55:400-9. doi: 10.1002/ana.10848. PubMed PMID: 14991818.

22. Hsu WY, Cheng CH, Liao KK, Lee IH, Lin YY. Effects of repetitive transcranial magnetic stimulation on motor functions in patients with stroke: 
a meta-analysis. Stroke. 2012;43:1849-57. doi: 10.1161/STROKEAHA.111.649756. PubMed PMID: 22713491.

23. Munoz Maniega S, Cvoro V, Chappell FM, Armitage PA, Marshall I, Bastin ME, et al. Changes in NAA and lactate following ischemic stroke: a serial MR spectroscopic imaging study. Neurology. 2008;71:1993-9. doi: 10.1212/01. wnl.0000336970.85817.4a. PubMed PMID: 19064881.

24. Cramer SC. Repairing the human brain after stroke. II. Restorative therapies. Ann Neurol. 2008;63:549-60. doi: 10.1002/ana.21412. PubMed PMID: 18481291.

25. Kim C, Choi HE, Jung H, Lee BJ, Lee KH, Lim YJ. Comparison of the Effects of $1 \mathrm{~Hz}$ and $20 \mathrm{~Hz}$ rTMS on Motor Recovery in Subacute Stroke Patients. Ann Rehabil Med. 2014;38:585-91. doi: 10.5535/ arm.2014.38.5.585. PubMed PMID: 25379487. PubMed PMCID: PMC4221386.

26. Desrosiers J, Bravo G, Hebert R, Dutil E, Mercier L. Validation of the Box and Block Test as a measure of dexterity of elderly people: reliability, validity, and norms studies. Arch Phys Med Rehabil. 1994;75:751-5. PubMed PMID: 8024419.

27. Mathiowetz V, Volland G, Kashman N, Weber K. Adult norms for the Box and Block Test of manual dexterity. Am J Occup Ther. 1985;39:386-91. PubMed PMID: 3160243.

28. Duncan PW, Propst M, Nelson SG. Reliability of the Fugl-Meyer assessment of sensorimotor recovery following cerebrovascular accident. Phys Ther. 1983;63:1606-10. PubMed PMID: 6622535.

29. Sullivan KJ, Tilson JK, Cen SY, Rose DK, Hershberg J, Correa A, et al. Fugl-Meyer assessment of sensorimotor function after stroke: standardized training procedure for clinical practice and clinical trials. Stroke. 2011;42:427-32. doi: 10.1161/ STROKEAHA.110.592766. PubMed PMID: 21164120.

30. Gladstone DJ, Danells CJ, Black SE. The fuglmeyer assessment of motor recovery after stroke: a critical review of its measurement properties. Neurorehabil Neural Repair. 2002;16:232-40. doi: 10.1177/154596802401105171. PubMed PMID: 12234086.

31. Wassermann EM. Risk and safety of repetitive transcranial magnetic stimulation: report and suggested guidelines from the International Workshop on the Safety of Repetitive Transcranial Magnetic Stimulation, June 5-7, 1996. Electroencephalogr Clin Neurophysiol. 1998;108:1-16. PubMed PMID: 9474057.
32. Yozbatiran N, Alonso-Alonso M, See J, Demirtas-Tatlidede A, Luu D, Motiwala RR, et al. Safety and behavioral effects of high-frequency repetitive transcranial magnetic stimulation in stroke. Stroke. 2009;40:309-12. doi: 10.1161/ STROKEAHA.108.522144. PubMed PMID: 18845801. PubMed PMCID: PMC3366156.

33. Kim YH, You SH, Ko MH, Park JW, Lee KH, Jang $\mathrm{SH}$, et al. Repetitive transcranial magnetic stimulation-induced corticomotor excitability and associated motor skill acquisition in chronic stroke. Stroke. 2006;37:1471-6. doi: 10.1161/01. STR.0000221233.55497.51. PubMed PMID: 16675743.

34. Ji S-G, Cha H-G, Kim M-K. Stroke recovery can be enhanced by using repetitive transcranial magnetic stimulation combined with mirror therapy. Journal of Magnetics. 2014;19:28-31. doi: 10.4283/ jmag.2014.19.1.028.

35. Rajak B, Gupta M, Bhatia D, Mukherjee A. Effect of repetitive Transcranial Magnetic Stimulation on hand function of spastic cerebral palsy children. J Neurol Disord. 2017;5:2. doi: 10.4172/23296895.1000339.

36. Niimi M, Hashimoto K, Kakuda W, Miyano S, Momosaki R, Ishima $\mathrm{T}$, et al. Role of Brain-Derived Neurotrophic Factor in Beneficial Effects of Repetitive Transcranial Magnetic Stimulation for Upper Limb Hemiparesis after Stroke. PLOS One. 2016;11:e0152241. doi: 10.1371/journal. pone.0152241. PubMed PMID: 27007747.PubMed PMCID: PMC4805302.

37. Zhang X, Mei Y, Liu C, Yu S. Effect of transcranial magnetic stimulation on the expression of $\mathrm{C}-\mathrm{Fos}$ and brain-derived neurotrophic factor of the cerebral cortex in rats with cerebral infarct. $J$ Huazhong Univ Sci Technolog Med Sci. 2007;27:415-8. doi: 10.1007/s11596-007-0416-3. PubMed PMID: 17828499.

38. Baba T, Kameda M, Yasuhara T, Morimoto T, Kondo A, Shingo T, et al. Electrical stimulation of the cerebral cortex exerts antiapoptotic, angiogenic, and anti-inflammatory effects in ischemic stroke rats through phosphoinositide 3-kinase/Akt signaling pathway. Stroke. 2009;40:e598-e605. doi: 10.1161/strokeaha.109.563627.

39. Gao F, Wang S, Guo Y, Wang J, Lou M, Wu J, et al. Protective effects of repetitive transcranial magnetic stimulation in a rat model of transient cerebral ischaemia: a microPET study. Eur J NuCl Med Mol Imaging. 2010;37:954-61. doi: 10.1007/ s00259-009-1342-3. PubMed PMID: 20107794.

40. Yoon KJ, Lee YT, Han TR. Mechanism of function- 
al recovery after repetitive transcranial magnetic stimulation (rTMS) in the subacute cerebral ischemic rat model: neural plasticity or anti-apoptosis? Exp Brain Res. 2011;214:549-56. doi: 10.1007/ s00221-011-2853-2. PubMed PMID: 21904929.

41. Ogiue-Ikeda M, Kawato S, Ueno S. Acquisition of ischemic tolerance by repetitive transcranial magnetic stimulation in the rat hippocampus. Brain Res. 2005;1037:7-11. doi: 10.1016/j. brainres.2004.10.063. PubMed PMID: 15777747.

42. Khedr EM, Etraby AE, Hemeda M, Nasef AM, Razek AA. Long-term effect of repetitive transcranial magnetic stimulation on motor function recovery after acute ischemic stroke. Acta Neurol Scand. 2010;121:30-7. doi: 10.1111/j.16000404.2009.01195.x. PubMed PMID: 19678808.

43. Greenough WT, Larson JR, Withers GS. Effects of unilateral and bilateral training in a reaching task on dendritic branching of neurons in the rat motor-sensory forelimb cortex. Behav Neural Biol. 1985;44:301-14. PubMed PMID: 2415103.

44. Jones TA, Chu CJ, Grande LA, Gregory AD. Motor skills training enhances lesion-induced structural plasticity in the motor cortex of adult rats. J Neuro- sci. 1999;19:10153-63. PubMed PMID: 10559423.

45. Kleim JA, Vij K, Ballard DH, Greenough WT. Learning-dependent synaptic modifications in the cerebellar cortex of the adult rat persist for at least four weeks. J Neurosci. 1997;17:717-21. PubMed PMID: 8987793.

46. Biernaskie J, Corbett D. Enriched rehabilitative training promotes improved forelimb motor function and enhanced dendritic growth after focal ischemic injury. J Neurosci. 2001;21:5272-80. PubMed PMID: 11438602.

47. Stroemer RP, Kent TA, Hulsebosch CE. Neocortical neural sprouting, synaptogenesis, and behavioral recovery after neocortical infarction in rats. Stroke. 1995;26:2135-44. PubMed PMID: 7482662.

48. Carmichael ST. Cellular and molecular mechanisms of neural repair after stroke: making waves. Ann Neurol. 2006;59:735-42. doi: 10.1002/ana.20845. PubMed PMID: 16634041.

49. Dobkin BH. Training and exercise to drive poststroke recovery. Nat Clin Pract Neurol. 2008;4:7685. doi: 10.1038/ncpneuro0709. PubMed PMID: 18256679. PubMed PMCID: PMC4099052. 